\title{
Paraplegia
}

\section{Functional Electrical Stimulation and Reciprocating Gait Orthosis for Ambulation Exercise in a Tetraplegic Patient: A Case Study}

\author{
C. A. Phillips, MD, PE, D. M. Hendershot, MS \\ Department of Biomedical and Human Factors Engineering, Wright State University, \\ Dayton, Ohio 45435, USA.
}

\section{Summary}

The purpose of this paper is to report the cardiopulmonary stresses (as indicated by heart rate, blood pressure, oxygen consumption and task cost) for a tetraplegic individual when walking with electrical stimulation and a gait orthosis as opposed to walking with a gait orthosis alone. Functional electrical stimulation (FES), when interfaced with a reciprocating gait orthosis $(R G O)$, resulted in walking exercise for a $C-7$ level tetraplegic subject, who was 8 years post-injury. Cardiopulmonary measurements were made and task costs calculated on this individual during progressive velocity walking with the FES-RGO and the orthosis alone (without FES). Results for the tetraplegic individual indicate that there were no significant changes in heart rate at the various walking velocities with respect to the two systems. However, systolic blood pressure was significantly reduced at the three highest walking velocities $(1 \cdot 2,1 \cdot 6$ and $2 \cdot 0 \mathrm{kph})$ when using the FES and orthosis system as compared to orthosis-alone walking $(p<\cdot 025$, $p<\cdot 005$ and $p<\cdot 025$ respectively). Calculated oxygen consumption was significantly reduced and calculated task cost improved at the highest walking velocity $(2 \cdot 0 \mathrm{kph})$ when using the FES-RGO system as compared to using only the RGO $(p<\cdot 025)$. It is concluded that cardiopulmonary stresses are significantly less for this tetraplegic individual when ambulating at the higher velocities with electrical stimulation and a gait orthosis than when walking with a gait orthosis alone. This may result in improved endurance during each walking session and (consequently) an improved aerobic exercise effect. This might also allow some tetraplegics, who would be unable to walk with the $R G O$ alone, to walk with electrical stimulation and the RGO.

Key words: Electrical stimulation; Gait orthosis; Physical therapy; Cardiopulmonary effects; Tetraplegia.

The reciprocating gait orthosis ( $\mathrm{RGO}$ ) has been employed for ambulation of patients with neuromuscular disorders (Douglas et al., 1984; McCall et al., 1983; Yngve et al., 1984). The RGO is a bilateral hip-knee-ankle-foot orthosis (HKAFO) 
with a pelvic band and thoracic support (for high-level paraplegics and tetraplegics).

FES may be applied during the swing phase of gait in combination with the RGO in order to assist the forward motion of the spinal cord injured individual. This was initially reported for paraplegic subjects (Petrofsky et al., 1985; 1986) and later reported for a tetraplegic subject (Phillips, 1989a; 1989b).

The purpose of this paper was to report the cardiopulmonary stresses (as reflected by heart rate, blood pressure, oxygen consumption and task cost) for a tetraplegic individual when walking with electrical stimulation and a gait orthosis versus walking with a gait orthosis alone.

\section{Methods}

A spinal cord injured individual participated in this study. G.B. is a 28-year-old, male, C-7 level tetraplegic who was injured 8 years ago. He sustained an incomplete injury with some sensation and slight (but functionally useless) movement below the level of injury. GB ambulated in an FES-RGO system described previously (Petrofsky et al., 1985; Phillips, 1989a).

\section{Progressive velocity ambulation}

The subject participated in an 8 month training protocol in which he used electrical stimulation and an orthosis to ambulate on a 5-day per week basis. The subject would perform forward walking for a 65 minute interval divided into:

(a) An initial 30 minute period

(b) A 5 minute rest period

(c) A final 30 minute period

Walking began at a baseline rate of $0.4 \mathrm{kph}$ and progressed in increments of 0.4 $\mathrm{kph}$ (i.e., $0 \cdot 4,0.8,1.2,1.6$ and $2.0 \mathrm{kph}$ ). Ambulation at a constant velocity was obtained by having the subject traverse a given distance (e.g., 1/10 kilometer) and simultaneously check the elapsed time with a stop watch. For a particular target velocity, the required elapsed time was precalculated from lap distance divided by target velocity. If the subject accomplished the lap at greater than or less than the calculated lap time, he would then be requested to increase or decrease his cadence (respectively) on the next lap. By successive approximations, the subject could consistently achieve a target velocity within plus or minus $5 \%$. A sensory feedback system for constant velocity control of RGO and FES-RGO ambulation exercise has subsequently been developed and is currently under evaluation (Phillips et al., 1990).

The walking involved progressive velocities. If the subject completed the initial 30 minute period at a given rate, he then proceeded to walk at the rate plus $0.4 \mathrm{kph}$ during the final 30 minute period. If the subject did not complete the initial 30 minute period at a given rate, he then proceeded to walk at that rate minus $0.4 \mathrm{kph}$ during the final 30 minute period. The two 30 minute back-to-back walking sessions allowed convenient scheduling of the subject.

Depending on whether the subject did or did not complete the final 30 minute period, the next session's initial velocity was calculated as the final 30 minute 
period's velocity plus or minus (respectively) $0.4 \mathrm{kph}$. In this manner, the subject underwent progressive velocity walking.

\section{Progressive velocity stress testing}

At least once (and usually twice) per month the subject participated in two progressive velocity stress tests. Initially, he ambulated at a velocity of $0.4 \mathrm{kph}$ for a 4 minute period. For 30 seconds thereafter (and while still walking), expired air was collected and the cardiopulmonary system analysed as described below. If the subject successfully completed the 4 minute walking period, there was a 5 minute rest period (to avoid cumulative fatigue effects). This was followed by another 4 minute walking period with the velocity incremented by $0.4 \mathrm{kph}$. After each 4 minute walking period expired, air was collected and analysed. There was then another 5 minute rest period. This protocol continued until the subject attained a velocity at which he could not complete the 4 minute walking period.

The two progressive velocity stress tests were done on separate days. One test employed electrical stimulation and a gait orthosis, and the other test was with the gait orthosis alone (hereafter referred to as with FES and without FES, respectively).

\section{Cardiovascular measurements}

At the conclusion of the 30 second expired gas collection interval, the subject stood erect, but was no longer walking. The systolic and diastolic blood pressures were then determined by manual sphygmomanometry and auscultation of the right arm. Simultaneously, another assistant determined the heart rate by manual palpation of the radial pulse of the left arm.

\section{Pulmonary Measurements and Task Cost}

The ventilation equivalent $\left(\overrightarrow{\mathrm{V}}_{\mathrm{E}}\right)$ and oxygen consumption $\left(\mathrm{V}_{2}\right)$ were determined as follows. The 30 seconds of expired air $\left(\mathrm{CO}_{2}\right.$ and unused $\left.\mathrm{O}_{2}\right)$ was sampled using blood gas syringes and expelled directly into the Beckman Medical Gas Analyser (Model $7000 \mathrm{IV}$ ), where $\mathrm{O}_{2}$ and $\mathrm{O}_{2}$ percentages by volume were calculated for the number of breaths that were expired per 30 second unit of time $\left(\dot{V}_{E}\right.$ in liters per half minute). The correction factor (for sea-level barometric pressure and body temperature) was determined by a standard chart using barometric pressure $(\mathrm{mmHg})$ and temperature $\left({ }^{\circ} \mathrm{C}\right)$. The corrected $\dot{\mathrm{V}}_{\mathrm{E}}$ was obtained by multiplying the correction factor by 1.44 and by two times the $\dot{V}_{E}$ (so that the two 30 second time periods would equal one minute). The $\mathrm{VO}_{2}$ was obtained by taking the corrected $\dot{\mathrm{V}}_{\mathrm{E}}$ times the percent true $\mathrm{O}_{2}$ and dividing by 100 . $\mathrm{VO}_{2}$ was the oxygen uptake rate and was calculated as follows:

$$
\mathrm{VO}_{2}=\frac{\left(\text { Cor. } \mathrm{V}_{\mathrm{E}}\right)\left(\% \text { true } \mathrm{O}_{2}\right)}{(100)}
$$

and

$$
\text { Cor. } \dot{\mathrm{V}}_{\mathrm{E}}=(2)\left(\dot{\mathrm{V}}_{\mathrm{E}}\right) \text { (Cor. Factor) }(1 \cdot 44)
$$

where: $\dot{V}_{E}$ is in liters per half minute.

Task cost is an index of cardiopulmonary performance. It is the ratio of useful work output (in kilograms times meters) for each milliliter of oxygen consumed. 
The index was developed by Pierrynowski, et al. (1981) and may be considered somewhat similar to 'miles per gallon' as an index of automobile performance: the higher the index, the better the performance.

The overall equation is:

$\frac{\mathrm{kg} \cdot \mathrm{m}}{\mathrm{ml} \mathrm{O}_{2}}=\frac{[\mathrm{wt}(\mathrm{kg})][\mathrm{vel}(\mathrm{km} / \mathrm{hr})][\cdot 0167 \mathrm{hr} / \mathrm{min})][1000(\mathrm{~m} / \mathrm{km})]}{\left[\hat{\mathrm{VO}}_{2}(\mathrm{ml} / \mathrm{min})\right]}$

Note that $\mathrm{VO}_{2}$ was calculated from eq. (1) and was determined with FES and without FES as described previously. The weight (in kilograms) represents the external weight of the system being utilised and the weight of the subject. This consisted of the gait orthosis and the walker (total of $5.45 \mathrm{~kg}$ ) and the weight of the patient $(66.55 \mathrm{~kg}$ ) for a total weight of $72.0 \mathrm{~kg}$. The expected (normal) value for a $70 \mathrm{~kg}$ person walking $3 \mathrm{kph}$ (assuming a 6 liter/minute cardiac output and $\mathrm{A}-\mathrm{V} \mathrm{O}_{2}$ difference of $6 \mathrm{ml} \mathrm{O} / 100 \mathrm{ml}$ ) would be about $10 \mathrm{~kg} \cdot \mathrm{m} / \mathrm{ml} \mathrm{O}_{2}$.

For this particular individual and system, the more simplified equation becomes:

$\frac{\mathrm{kg} \cdot \mathrm{m}}{\mathrm{ml} \mathrm{O} \mathrm{O}_{2}}=\frac{\operatorname{vel}\left(\frac{\mathrm{km}}{\mathrm{hr}}\right) \cdot 1200\left(\frac{\mathrm{kg} \cdot \mathrm{m} \cdot \mathrm{hr}}{\mathrm{km} \cdot \mathrm{min}}\right)}{\mathrm{VO}_{2}\left(\frac{\mathrm{ml} \mathrm{O}}{\mathrm{min}}\right)}$

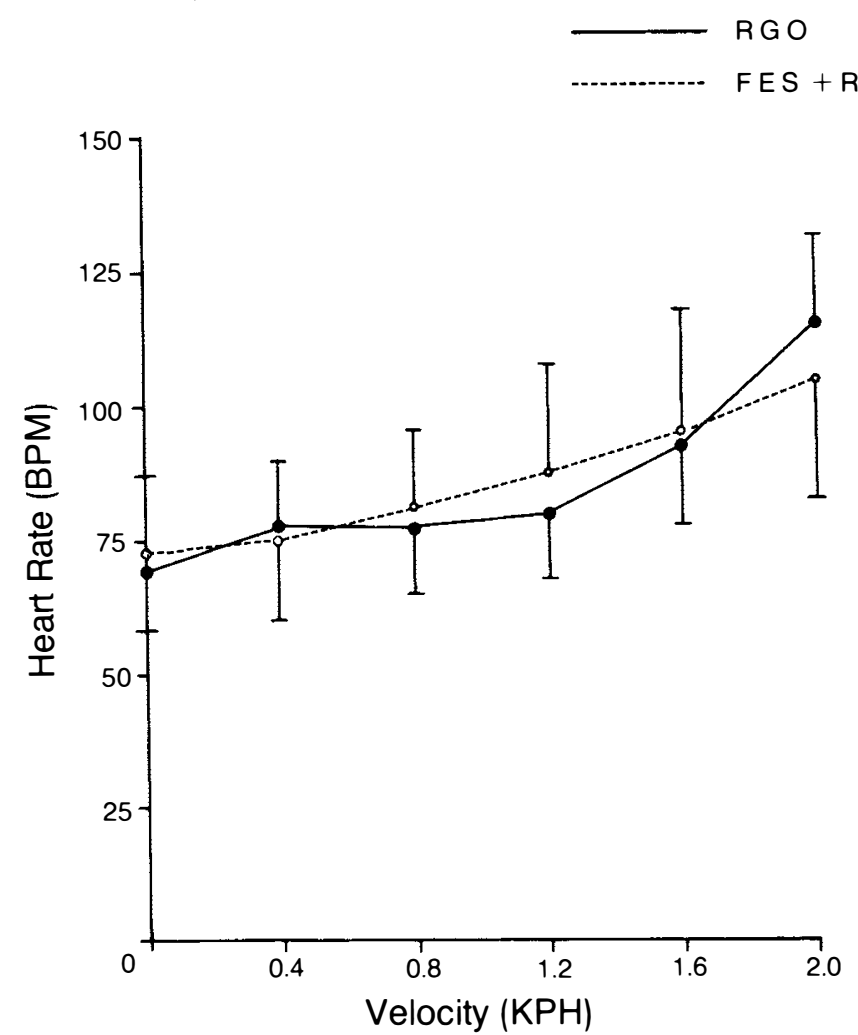

Figure 1 Heart rate as a function of ambulation velocity with the FES-RGO and with the RGO alone. 


\section{Results}

Figure 1 indicates the measured heart rate as a function of ambulation velocity using just the gait orthosis and walker (without FES) and using the system (with FES). All data in Fig 1 through to 5 were acquired over the 8 month protocol and were obtained when the preferred walking velocity was $1.6 \mathrm{kph}$.

Figure 2 shows the measured systolic blood pressure as it relates to ambulation velocity using the RGO alone and using the RGO (with FES). Note that systolic blood pressure is significantly reduced when ambulating with FES and the orthosis (compared to orthosis alone) at the higher velocities of $1.2 \mathrm{kph}(\mathrm{p}<\cdot 025), 1.6 \mathrm{kph}$ $(\mathrm{p}<\cdot 005)$ and $2 \cdot 0 \mathrm{kph}(\mathrm{p}<\cdot 025)$.

Figure 3 shows the measured diastolic blood pressure as a function of ambulation velocity using only the RGO and also with FES and the RGO. There is no significant difference between the two systems.

Figure 4 relates the calculated oxygen consumption as a function of ambulation velocity using the gait orthosis and walker (without FES) and also using the FES and orthosis system. At the highest velocity of ambulating $(2 \cdot 0 \mathrm{kph})$, the oxygen consumption is significantly reduced when walking with FES and the RGO when compared to RGO walking alone $(\mathrm{p}<\cdot 025)$.

Figure 5 reports the calculated task cost as a function of ambulation velocity

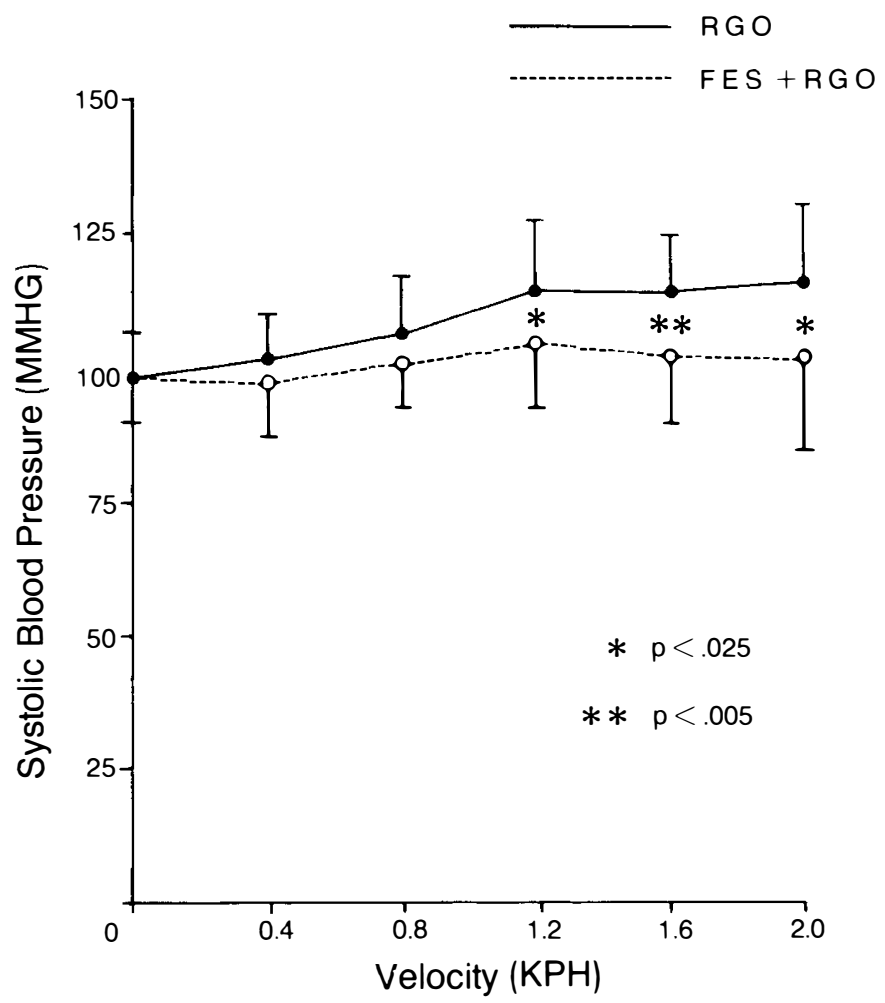

Figure 2 Systolic blood pressure as a function of ambulation velocity with the FES-RGO and with the RGO alone. 


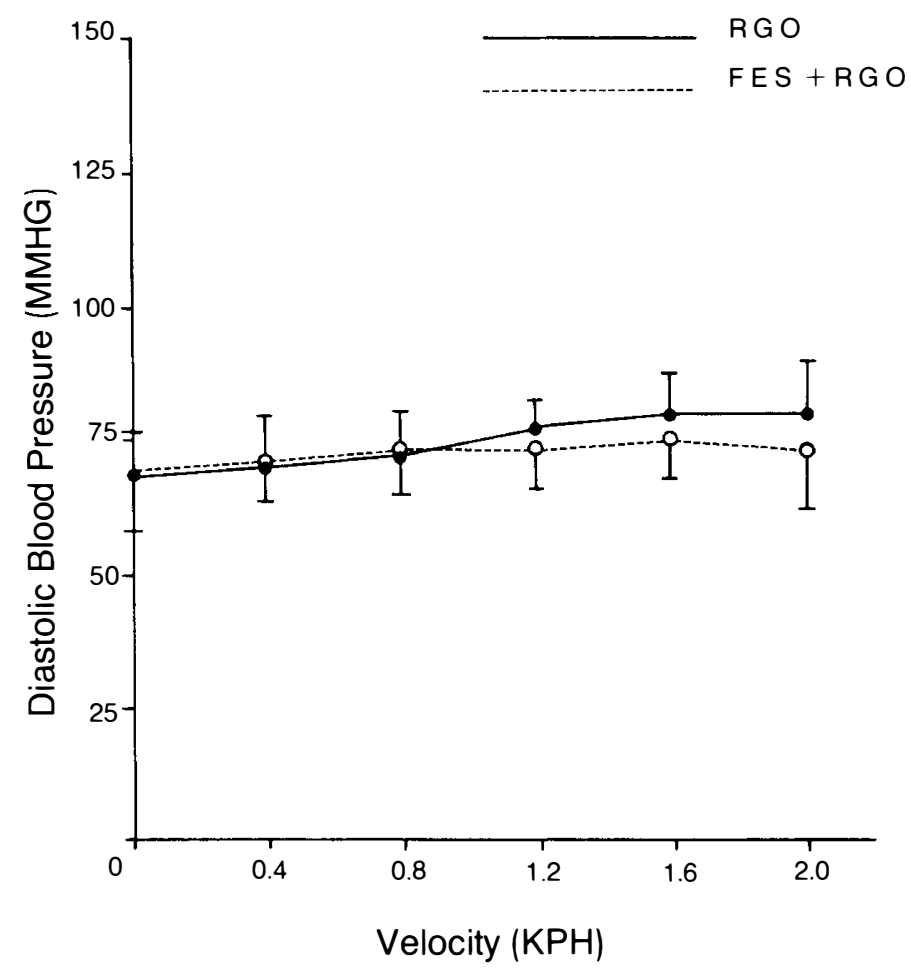

Figure 3 Diastolic blood pressure as a function of ambulation velocity with the FES-RGO and with the RGO alone.

using just the gait orthosis and walker (but no FES) as compared to using the FES and orthosis system. Statistical analysis indicates that the task cost of walking with FES and the RGO is significantly improved at the highest walking velocity $(2 \cdot 0$ $\mathrm{kph})$ as compared to walking with just the RGO $(\mathrm{p}<\cdot 025)$.

\section{Discussion}

The ambulation described above provides exercise that is actually only one phase in a comprehensive program of FES physical therapy. With FES, the functionally electrically stimulated muscles act upon their environment to produce the exercise effect. This is in contrast to conventional physical therapy (no FES) in which the environment (e.g. the physical therapist) acts upon the muscle to produce the exercise effect (Phillips et al., 1984). Phillips (1987) defined functional electrical rehabilitation to consist of: (1) an isokinetic leg trainer; (2) a stationary bicycle ergometer; and (3) an FES-orthosis for ambulation exercise. The subject for this study preconditioned for 6 months using the first two exercise modalities prior to proceeding to ambulation exercise with the FES-RGO.

Although there was no significant difference in heart rate when using the two systems, higher heart rates (above $100 \mathrm{BPM}$ ) were found at the higher walking velocities. However, the overall variation is rather wide. In tetraplegics, 


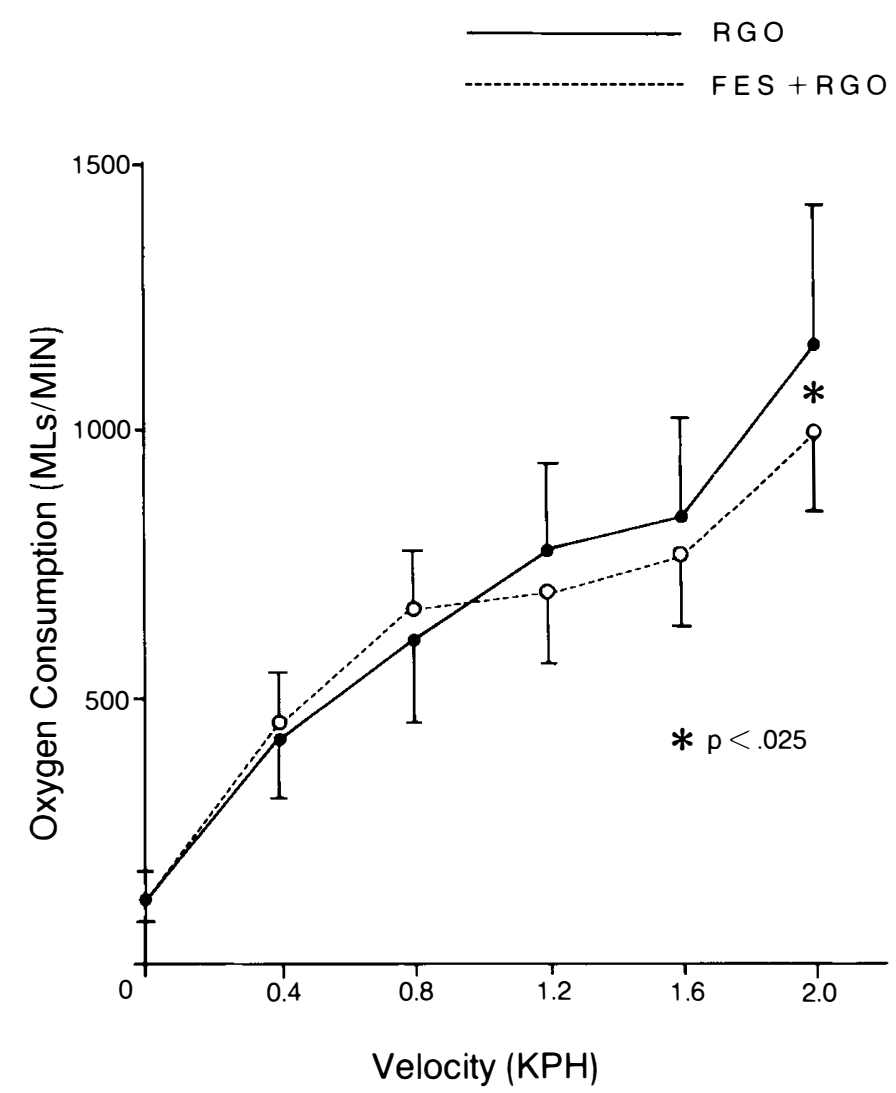

Figure 4 Oxygen consumption as a function of ambulation velocity with the FES-RGO and with the RGO alone.

sympathetic contribution to the cardiac plexus is damaged and hence cardiovascular response to physical stress can be unpredictable. It also has been stated by Knutsson et al. (1973) that in cervical cord transection, heart rate regulation is attained by varying vagal tone. The heart rate increase in these subjects is restricted up to levels just above $100 \mathrm{BPM}$. In this study the rates were somewhat higher, but the subject had an incomplete lesion (consistent with Frankel class $\mathrm{C}$ ) as opposed to a complete lesion (Frankel class A). Therefore, our results cannot be directly compared with those of Knutsson et al. (1973).

It was noted that the subject's preferred walking velocity was approximately $1 \cdot 6$ $\mathrm{kph}$. This was so whether only the RGO was utilised or the combination of FES and RGO. This is quite consistent with the task cost data (Figure 5) since it represents the point at which task cost is maximised regardless of the system utilised. Simply stated, the subject was the most efficient at this particular walking velocity.

This is not to say, however, that the reduction in $\mathrm{VO}_{2}$ and the increase in task cost are not significant because they occurred at a higher walking velocity (rather than thje preferred walking velocity). The purpose of the porogressive velocity walking protocol (see Methods) was in fact to physically condition the subject by 

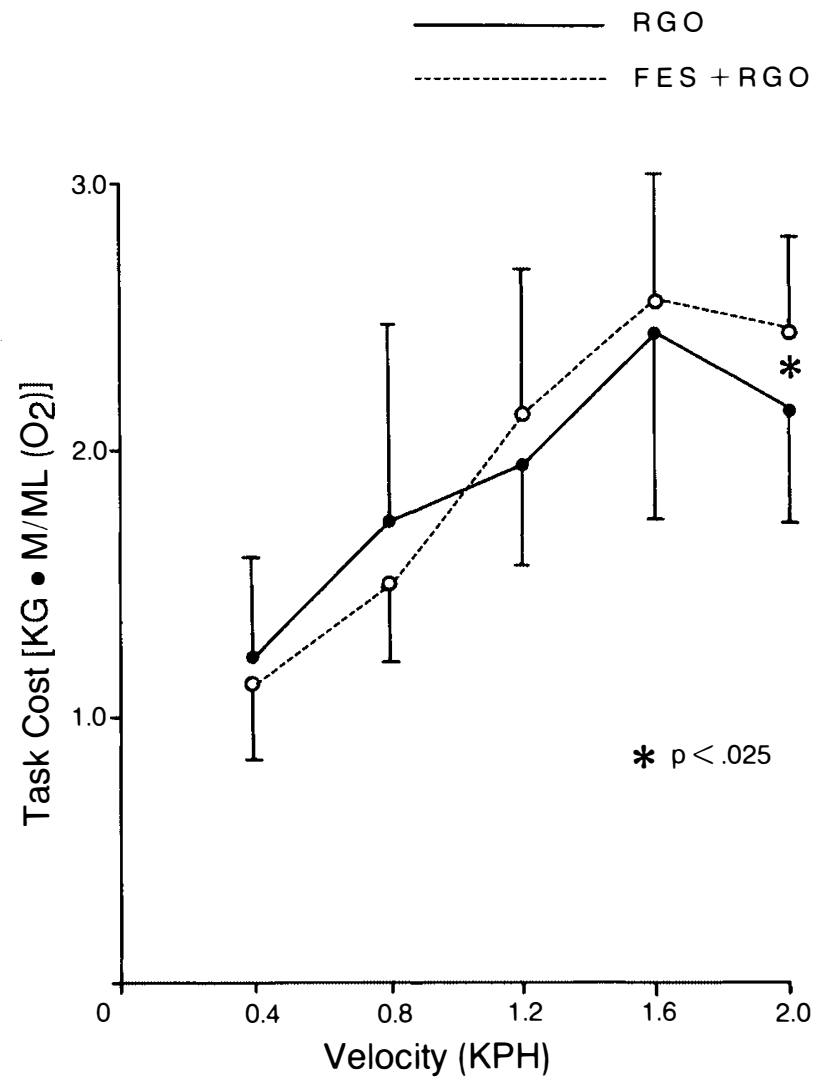

Figure 5 Task cost as a function of ambulation velocity with the FES-RGO and with the RGO alone.

continually training him to ambulate at the highest possible velocity. Therefore, the significance of reduced $\mathrm{VO}_{2}$ and increased task cost with an FES-RGO system as opposed to an RGO system alone (at the highest velocity) is that the former is the better system with respect to physical conditioning potential by providing a better aerobic training effect (vide infra).

This paper has provided specific information concerning the cardiopulmonary responses to ambulation exercise with the FES-RGO. Systolic blood pressure was significantly reduced when walking with FES and an orthosis (compared to orthosis alone) at the higher walking velocities of $1.2 \mathrm{kph}, 1.6 \mathrm{kph}$ and $2.0 \mathrm{kph}$. Moreover, at the highest walking velocity $(2.0 \mathrm{kph})$, the oxygen consumption was significantly reduced when walking with FES and the RGO when compared to RGO walking alone. Also, the task cost of walking with FES and the RGO is significantly improved at the highest walking velocity $(2.0 \mathrm{kph})$ as compared to walking with just the RGO.

The end result is that cardiopulmonary stresses are significantly less for this tetraplegic individual when ambulating at the higher velocities with electrical stimulation and a gait orthosis as opposed to ambulating with a gait orthosis alone. This finding should translate into improved endurance during each walking session 
(and result in longer walking times). Consequently, the aerobic exercise effect (e.g., muscular endurance training and improved cardiopulmonary reserve) should be enhanced when a quadriplegic walks with FES and an RGO instead of an RGO alone. This certainly is a testable hypothesis and will be the subject of a future study.

Finally, the lowered cardiopulmonary stresses at the higher velocities might allow some quadriplegics who would be unable to walk at a reasonable pace with the RGO alone, to walk with electrical stimulation and the RGO. One such case has been recently reported by Solomonow, et al. (1988). As electrical stimulation with orthotic bracing becomes more universally applied, additional case studies should particularly examine this specific issue.

\section{References}

Douglas R, Larsun P, D’Aubrosia R, McCall R 1983 LSU reciprocating gait orthosis. Orthopedics 6:834-839.

Knuttson E, Lewenhaupt-Olsson E, Thorsen M 1973 Physical work capacity and physical conditioning in paraplegic patients. Paraplegia 11:205-216.

McCall RE, Douglas R, Nicholas R 1983 Surgical treatment in patients with myelodysplasia before using the LSU reciprocating system. Orthopedics 6:843-848.

Petrofsky JS, Phillips CA, Douglas R, LARson P 1986 A computer-controlled walking system: The combination of an orthosis with functional electrical stimulation. F Clin Engrg 11:121-133.

Petrofsky JS, Phillips CA, Larson P, Douglas R 1985 Computer-synthesized walking-an application of orthosis and functional electrical stimulation (FES). Fournal of Neurological and Orthopedic Medical and Surgery 6:219-230.

PHILlIPS CA 1987 The medical criteria for active physical therapy: physician guidelines for patient participation in a program of functional electrical rehabilitation. American fournal of Physical Medicine 66:269-286.

PHILlips CA 1989a Functional electrical stimulation and lower extremity bracing for ambulation exercise of the S.C.I. individual: A medically prescribed system. Physical Therapy 69:842-849.

PHILlips CA 1989b An interactive system of electronic stimulators and gait orthosis for walking in the spinal cord injured. Automedica (Lond.), 11:247-261.

Phillips CA, Heard D, Hendershot D, Koubek R 1990 A sensory feedback system for control of constant gait velocity: Application for E.M.S.-R.G.O. exercise. Engrg. Med. Biol. Soc. 12th Annual International Conference 12:2260-2261.

Phillips CA, Petrofsky JS, Hendershot DM, Stafford D 1984 Functional electrical exercise: a comprehensive approach for physical conditioning of the spinal cord injured individual. Orthopedics 7:112-1123.

Pierrynowski MR, Winter DA, Norman RW 1981 Metabolic measures to ascertain the optimal load to be carried by man. Ergonomics 24:393-399.

Solomonow M, BARATTA R, SHOJi H et al. 1988 F.E.S. powered locomotion of paraplegics fitted with the L.S.U. R.G.O. I.E.E.E. Engrg. Med. Biol. Soc. 10th Ann Internat Conf, 10:1672.

YNGVE D, Douglas R, ROBERTS JM 1984 The reciprocation gait orthosis and myelomeningocele. Fournal of Pediatric Orthopedics 4:304-310. 